\title{
Application of an Electromyographic Index of Gait Instability to Developmental Process of Supported Walking in Normal Neonates and Infants
}

\author{
Hideaki Yamashita ${ }^{1)}$, Kayoko Окамото ${ }^{1)}$, Tsutomu Okamoto ${ }^{2)}$, Paul D. Andrew3), \\ RYOKEI OGAWA ${ }^{1)}$ \\ ${ }^{1)}$ Department of Orthopaedic Surgery, Kansai Medical University, Fumisono-cho 10-15, \\ Moriguchi-shi, Osaka 570-8506, Japan. TEL +81 6-6992-1001 \\ ${ }^{2}$ Department of Liberal Arts, Kansai Medical University \\ ${ }^{3}$ Institute of Health Sciences, Hiroshima University School of Medicine
}

\begin{abstract}
The purpose of this study was to see whether an electromyographic (EMG) index of gait instability is applicable to the developmental process of supported walking in normal neonates and infants. In six neonates ranging in age from 14 to 26 days after birth, EMGs of stepping were recorded at approximately from one to four week intervals until around four months. Additionally, longitudinalEMGs of one subject were recorded at one or two week intervals until just before independent walking. EMG patterns of the lateral gastrocnemius (an ankle plantarflexor) and vastus medialis (a knee extensor) in the latter part of swing phase indicating unstable walking, not seen in the neonatal period up to first postnatal month, tended to increase in young infants at around three postnatal months. These results suggest the addition of voluntary infant stepping to reflex neonate stepping from around three months. From six to twelve months, these marked activities tended to decrease, gradually coming to resemble adult stable walking through development of strength, balance, and postural control. In conclusion, muscular activities of the lateral gastrocnemius and vastus medialis in the latter part of swing phase indicate unstable walking, findings which are applicable to developmental changes during newborn stepping and infant supported walking.
\end{abstract}

Key words: Infant supported walking, Developmental process, EMG, Gait instability.

(This article was submitted Jul. 7, 1998, and was accepted May. 20, 1999)

\section{INTRODUCTION}

Human gait without support is associated with muscle strength, balance, and postural control ${ }^{1,2}$. Although some people have to resort to supported walking by virtue of immaturity, aging, postoperative status, or disease, supported walking generally resembles the normal adult walking pattern except for the substitution of missing postural and balance elements with support ${ }^{3-7)}$. However, to decide whether gait is stable or not is very difficult to evaluate from motion analysis alone, for example, by comparison with a normal pattern. Although supported walking may show no perceptible change during rehabilitation, the underlying EMG pattern may be changing during the recovery of gait $^{4)}$. To evaluate gait stability during supported walking, it is therefore very important examine muscle function.

Okamoto et al.5) have determined signs of unstable walking from EMG activity patterns based 
Table 1. EMG index of gait instability

\begin{tabular}{lllc}
\hline Joint & & Sign of instability & Interpretation \\
\hline Ankle & SW-LG & Activity in the LG during SW (+) & Unstable \\
Knee & SW-VM & Activity in the VM during SW (+) & Unstable \\
\hline
\end{tabular}

SW: latter part of swing phase, LG: lateral gastrocnemius, VM: vastus medialis. $(+)$ : noticeable activity.

on the developmental process of normal infant walking 3, 5-9) and normal adult walking $3,5,10,11$ ) (Table 1), and have used them to analyze gait in terms of development processes and also as a way to electromyographically assess clinical process in gait training. They have reported that the vastus medialis is active in the latter half of swing phase, the tibialis anterior and rectus femoris are active during stance phase, and activity of the vastus medialis is continuous, as seen in a child within the first month of learning to walk. These electromyographic characteristics are not usually seen in subsequent childhood gait or in adult gait, and they serve as markers of very unstable gait. Activity of the gastrocnemius in the latter half of swing phase is generally noted only within the first three months after the child learns to walk, and that activity is interpreted as a sign of unstable gait. Activity of the gastrocnemius in the first half of stance phase and continuous activities of the biceps femoris and gluteus maximus from initial contact with the floor until push off are found in children until three years of age. These activities are considered electromyographic markers of slightly unstable gait.

We were interested in applying the index of gait instability to the developmental process of supported walking in normal neonates and infants. Newborn stepping has been an object of study for a long time ${ }^{2,12-20)}$. McGraw ${ }^{12)}$ and Zelazo et al. ${ }^{16)}$ have discussed the significance of early stepping movements for development of adult gait. Only a few attempts so far, however, have been made to study developmental changes of stepping by EMG, particularly in relation to gait instability. In this study, we applied the idea of the index of gait instability5) to developmental changes during newborn stepping and infant supported walking.

\section{METHOD}

Four male and two female infants were observed at $14,18,19,22,23$, and 26 days after birth. Crite- ria used for selecting the subjects were that they be full-term with birth weight between $2500 \mathrm{~g}$ and $4200 \mathrm{~g}$. They were screened by pediatricians to rule out abnormalities and illnesses. Motor development of each subject was within normal limits.

EMGs of all subjects were recorded from the neonatal period (the first month of age) to the young infant period (from one to four months after birth) at intervals of approximately one to four weeks. To more closely examine the electromyographic developmental changes from newborn stepping to supported walking just before independent walking, EMGs of one of the six subjects were recorded 26 times, at one or two week intervals, from 22 days after birth to 351 days, before independent walking.

In order to induce newborn stepping, the examiner was instructed to hold an infant under the arms with the soles of the feet touching a horizontal flat surface. Well-coordinated walking movements were observed from birth to around three months. In this study, because we could not successfully determine stepping, especially when the infants were lively, crying, hungry, or slightly excited, we selected well-coordinated walking movements consisting of three or more steps (Fig. 1).

The EMGs were recorded from six muscles in the right leg: tibialis anterior (TA), lateral gastrocnemius (LG), vastus medialis (VM), rectus femoris (RF), biceps femoris (BF), and gluteus maximus (GM), and from four muscles in the left leg: the TA, LG, RF, and BF. Surface electrodes $5 \mathrm{~mm}$ in diameter were used (Fig. 1). To attenuate artifacts in the surface electrode recordings, skin impedance was lowered by scratching loci of the electrodes lightly with a needle before the electrodes were applied $^{21)}$. The EMG recordings were made with an 18-channel pen-writing electroencephalograph $(60 \mathrm{~mm} / \mathrm{sec})$ with the gain set at $12 \mathrm{~mm} / 0.5 \mathrm{mV}$. An analog pulse signal from the video recording camera (60 frames/sec) was recorded simultaneously with the EMGs, so that any frame of the 

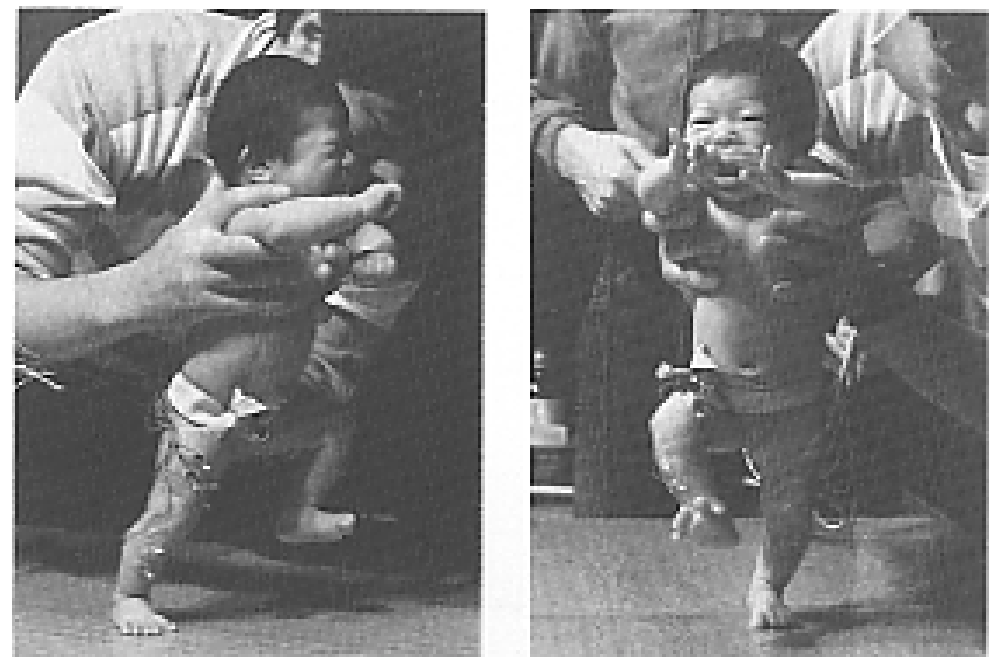

Fig. 1. Newborn stepping at 22 days after birth (subject A)

visual recording could be synchronized with the analog record. The walking cycle was divided into swing phase (SW) and stance phase (ST) by the video recordings.

\section{RESULTS}

We initially observed developmental process of supported walking in neonates and infants using an index of gait instability ${ }^{5)}$. In interpreting the EMG pattern of supported walking in all subjects tested, we focused on the discharge pattern in the latter part of swing phase and in stance phase. From this, we could see developmental changes in the EMG patterns of swing phase and relatively wide variations in those of stance phase. Most of the figures in this paper are from longitudinal representative EMG patterns in subject A (Fig. 1; 22 days after birth).

\section{1) Until first month of age}

Figure 2 shows representative EMG patterns of stepping at 22 days after birth. This is one of the more rhythmical walking-like patterns that we obtained in this neonate. The discharge patterns of leg muscles during newborn stepping are generally more irregular than in adult walking $3,5,10,11,15,19$ ).

The walking in this period was characterized by quick hip and knee flexion in which the thigh became almost horizontal in the first part of swing phase. The foot was raised forward and dorsiflexed strongly as shown in Fig. 1. Then the foot began to reach the floor slowly, the knee extending passively along with the hip. The foot usually contacted the floor with the heel first, but in few instances the forefoot made initial contact. A squatting posture was often observed during stance phase. At around one month, such squatting began to become less pronounced.

As the ankle and knee were extending, activities in the LG (an ankle plantarflexor) and VM (a knee extensor) were hardly seen in the second half of swing phase (Fig. 2). In stance phase continuous activities were seen in the ankle, knee, and hip muscles, especially in single stance. The discharge patterns of many leg muscles (TA, LG, VM, RF, $\mathrm{BF}$, and GM) indicated unstable walking5) showing reciprocal and cocontraction patterns, with wider variations and more excessive activity of discharge patterns than in adult walking $3,5,10,11,15,19)$. The TA and LG showed reciprocal patterns on the right and cocontraction on the left. Activity of the TA decreased or disappeared while that of the LG increased in many instances. Conversely, in some of the subjects activity of the LG decreased or disappeared while that of the TA increased. In the knee and hip muscles, the RF and BF showed both reciprocal and cocontraction patterns. In some subjects, the reciprocal pattern was one in which activity of the RF decreased or disappeared while that of the BF increased, whereas in other subjects the converse reciprocal pattern prevailed. Among 


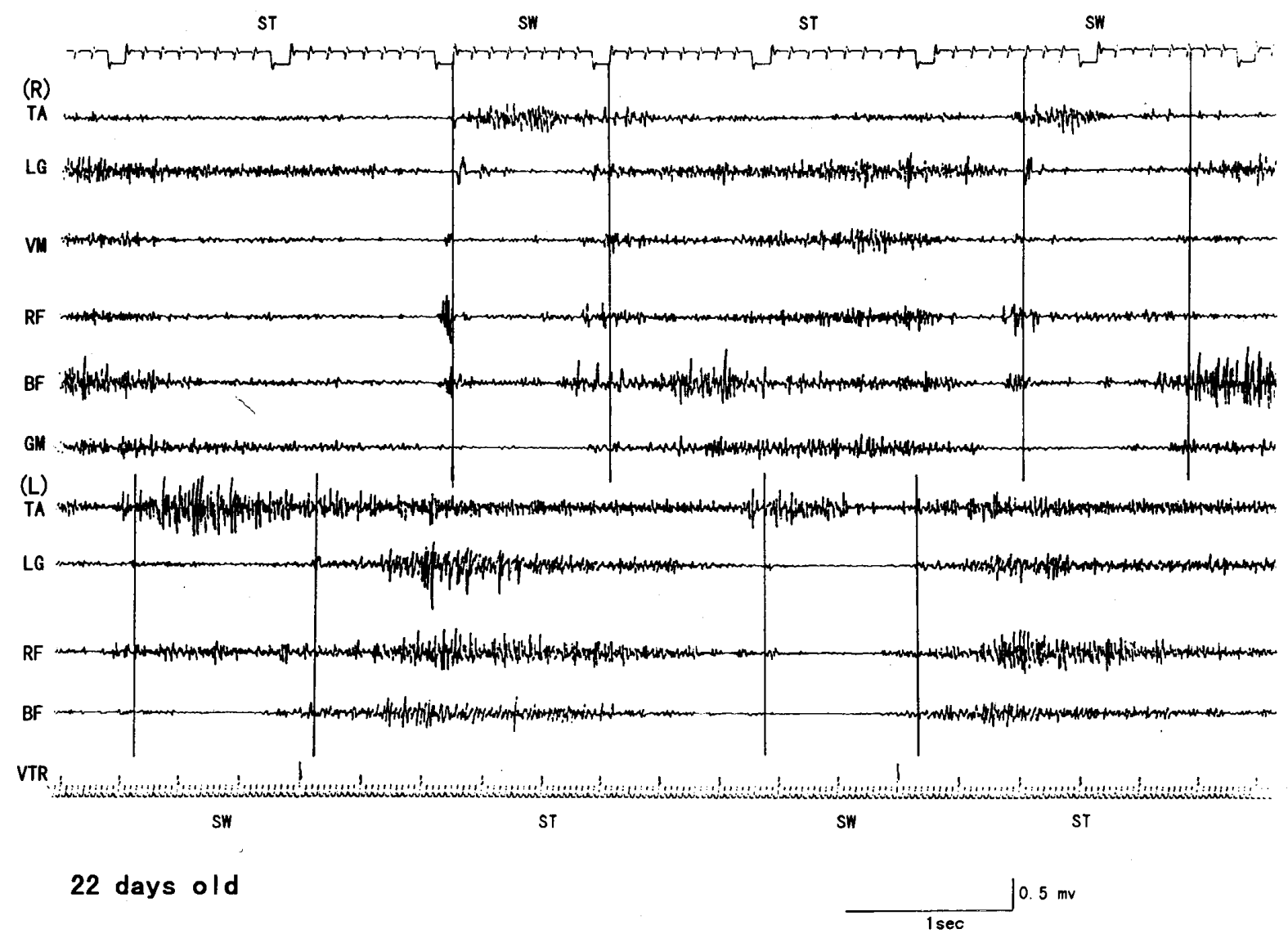

Fig. 2. EMGs of newborn stepping at 22 days after birth (same subject as in Fig. 1). ST: stance phase, SW: swing phase, (R): right leg, (L): left leg, TA: tibialis anterior, LG: lateral gastrocnemius, VM: vastus medialis, RF: rectus femoris, BF: biceps femoris, GM: gluteus maximus

the knee muscles, continuous activity of the VM was often observed, but sometimes no activity was seen at all.

\section{2) From one to four months of age}

Figures 3 and 4 show representative EMG patterns of stepping at 44 and 105 days after birth in the same infant. We found in six infants tested that we could induce a stepping pattern after one month of age, but not so easily as before one month.

After one month, leg flexion was performed strongly in the first part of swing phase as in the neonatal period, but the degree of hip flexion tended to decrease slightly. We found mostly plantarflexion before floor contact rather than dorsiflexion which was more prevalent in the newborn period. From three to four months, the toe initially contacted the floor for the most part. Knee extension was performed more actively than in the neonatal period. After around one month, a halfsquatting posture during stance phase tended to increase.

From one to three months, the LG and VM began to show activity before the foot actually touched the floor (Fig. 3). From three to four months, such activity become more pronounced (Fig. 4). On the other hand, we found wide variations in the discharge patterns of many leg muscles (TA, LG, VM, RF, BF, and GM) during stance phase (Figs. 3, 4), similar to the neonatal period (Fig. 2). The stepping did not cease at around four or five months after birth, as shown in the longitudinal observations of subject A.

\section{3) From six to twelve months of age}

During infant supported walking (6-12 months of age), step frequency was more regular than during the newborn period. The relatively pronounced 


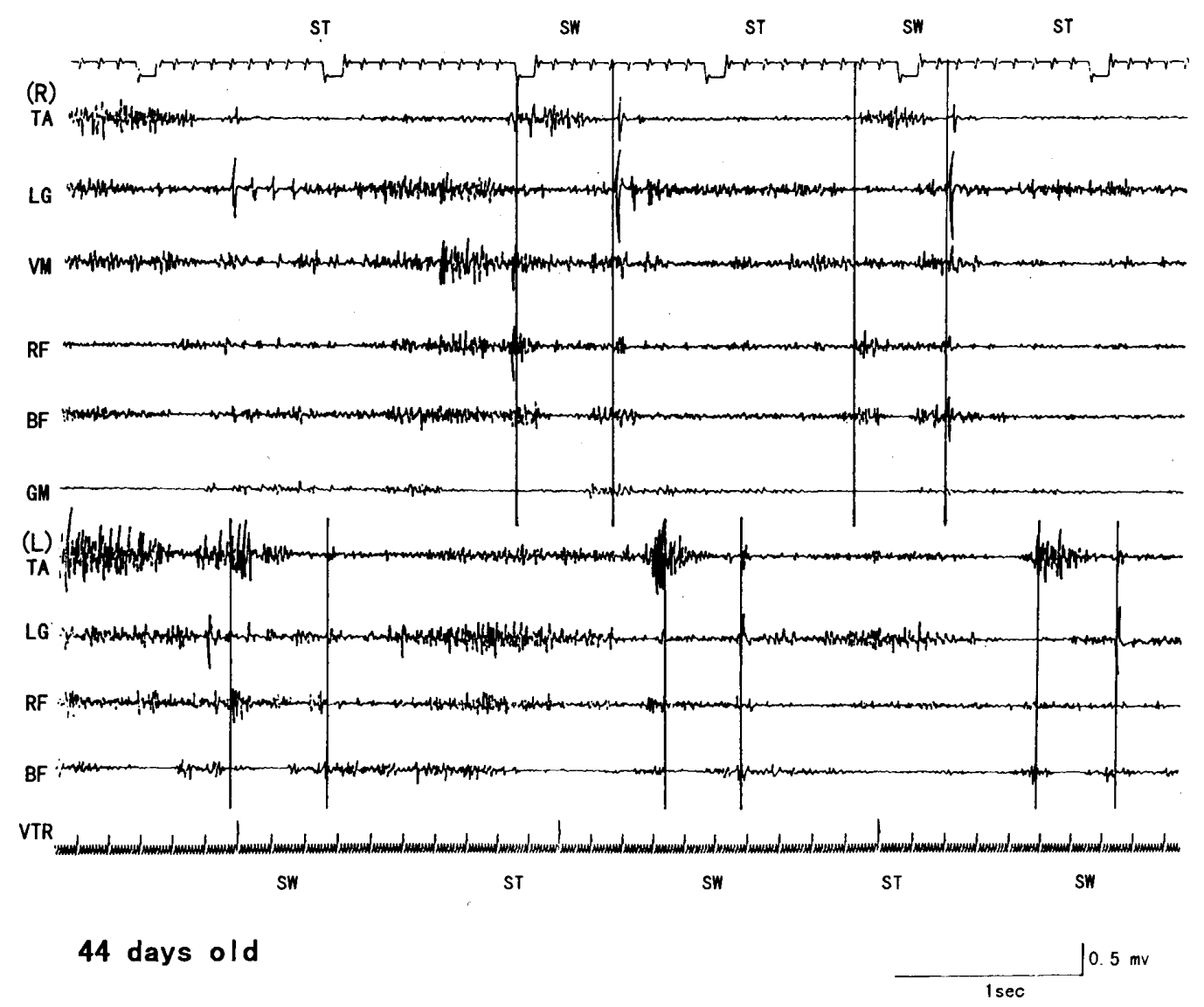

Fig. 3. EMGs of newborn stepping at 44 days after birth (same subject as in Figs. 1 and 2). Abbreviations are the same as those in Fig. 2.

flexion in hip and knee seen in the previous period was slightly reduced.

Figure 5 shows representative EMG patterns of supported walking at 351 days after birth, but before independent walking was achieved. From 11 to 12 months after birth, the infant became able to stand by herself and to walk with one-handed supported. The femur was not actively lifted up in the first part of swing phase and active ankle plantarflexion and knee extension tended to disappear in the latter part of swing phase. The heel usually contacted the floor first. This subject began to walk without support at 385 days after birth.

Strong myoelectric activity of the LG and VM seen in earlier periods (Figs. 3, 4) tended to disappear in the latter part of swing phase in this period (Fig. 5). The marked variations in the discharge patterns of both these muscles seen previously were no longer seen during infant supported walking, and more closely resembled adult walking 3,5 ,
10, 11, 15, 19). Cocontraction patterns of ankle, knee, and hip muscles seen in newborn stepping also tended to decrease or disappear during stance phase, but reciprocal patterns of mutual antagonists generally remained. Nevertheless we did find some instances of excessive muscular activity in many leg muscles during stance phase, similar to what was seen in earlier periods (Figs. 2, 3, 4).

\section{4) Developmental changes of EMG patterns}

Figure 6 shows the EMG patterns of ankle and knee extensors before foot contact during stepping at 14 and 83 days after birth (subject B), illustrating the differences between neonate and young infant periods.

In the neonatal period (up to 1 month), we could not see strong discharges of the LG and VM in the latter part of swing phase (Fig. 6, top). However, in the young infant period (1-4 months), we could see strong discharges of both these muscles in the 


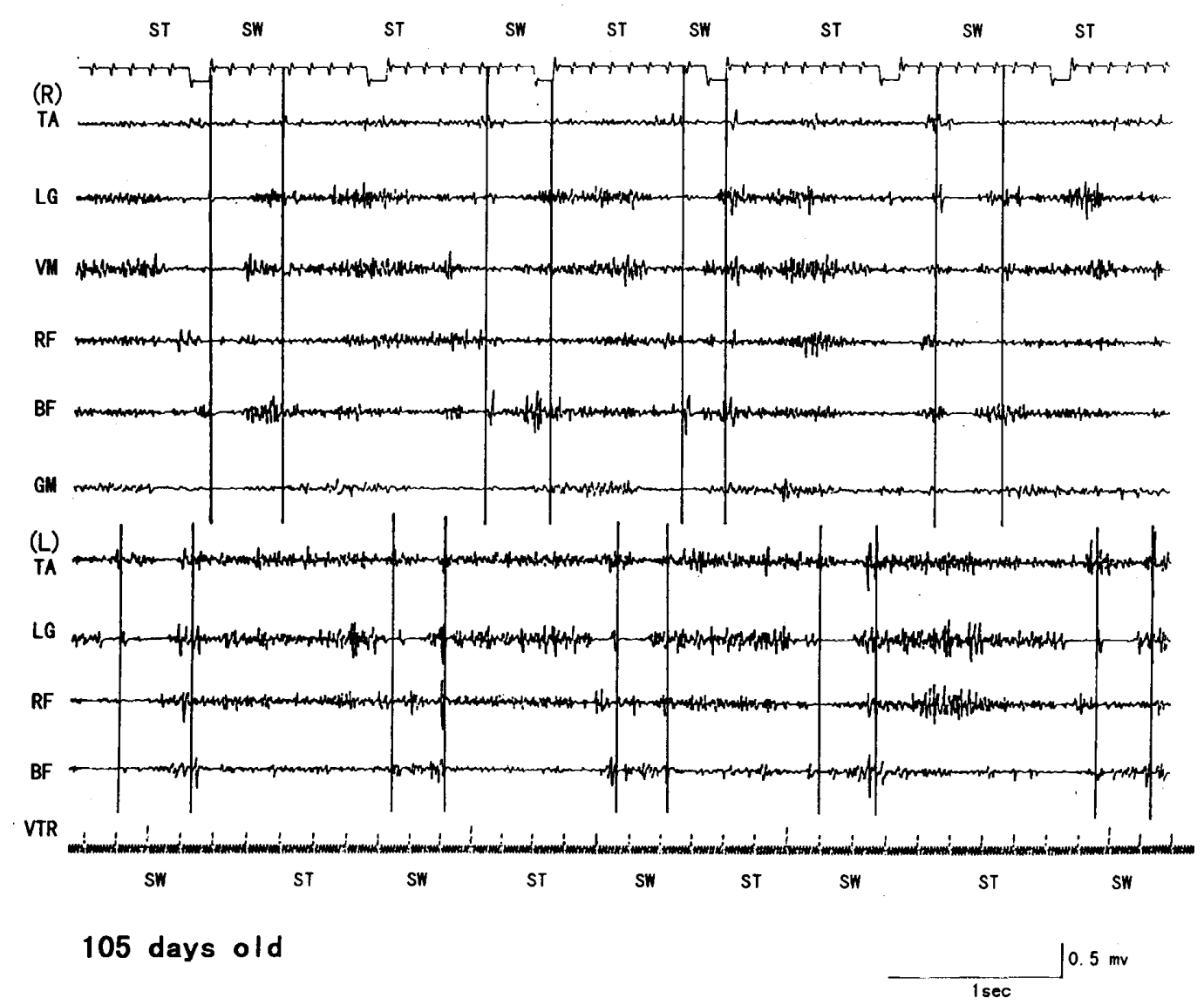

Fig. 4. EMGs of newborn stepping at 105 days after birth (same subject as in Figs. 1, 2, and 3). Abbreviations are the same as those in Fig. 2.

same phase in many instances, as mentioned above (Fig. 6, bottom). These developmental changes in muscle activity from the neonatal period to the young infant period were similar to those of subject A (Figs. 2, 3, 4).

\section{5) Application index of gait instability to supported walking in neonates and infants}

Table 1 shows the EMG index of gait instability in the latter part of swing phase that Okamoto et al. ${ }^{5)}$ have recently reported. Table 2 shows the results of evaluating developmental changes in LG and VM muscular activities during newborn stepping of each subject using the criteria for gait instability (Table 1). Table 3 shows developmental changes of muscular activities in the LG and VM before foot contact, from newborn stepping to supported walking just prior to independent walking in subject A. From the results obtained in the swing phase, it was discovered that we were able to apply the idea of the index of gait instability5) to developmental changes during newborn stepping and also infant supported walking.

\section{DISCUSSION}

McGraw ${ }^{12)}$ reported that infant stepping can be elicited shortly after birth and during the first months, and that thereafter it usually disappears. Thelen et al. ${ }^{2)}$, Forssberg ${ }^{15)}$, and Yamashita et al. ${ }^{19)}$ pointed out from movement patterns and EMGs that the locomotor pattern of the newborn differs markedly from that of an adult. Usually the leg muscle activities of newborn stepping are irregular and include a high degree of coactivation $2,14,17-19)$ compared with the adult walking pattern $3,5,10,11,15,19$ ).

We first interpret the meaning of wide variations in stance phase, especially in the EMG patterns of mutual antagonists of the leg muscles during stepping (Figs. 2, 3, 4). Diminution of activity in the 


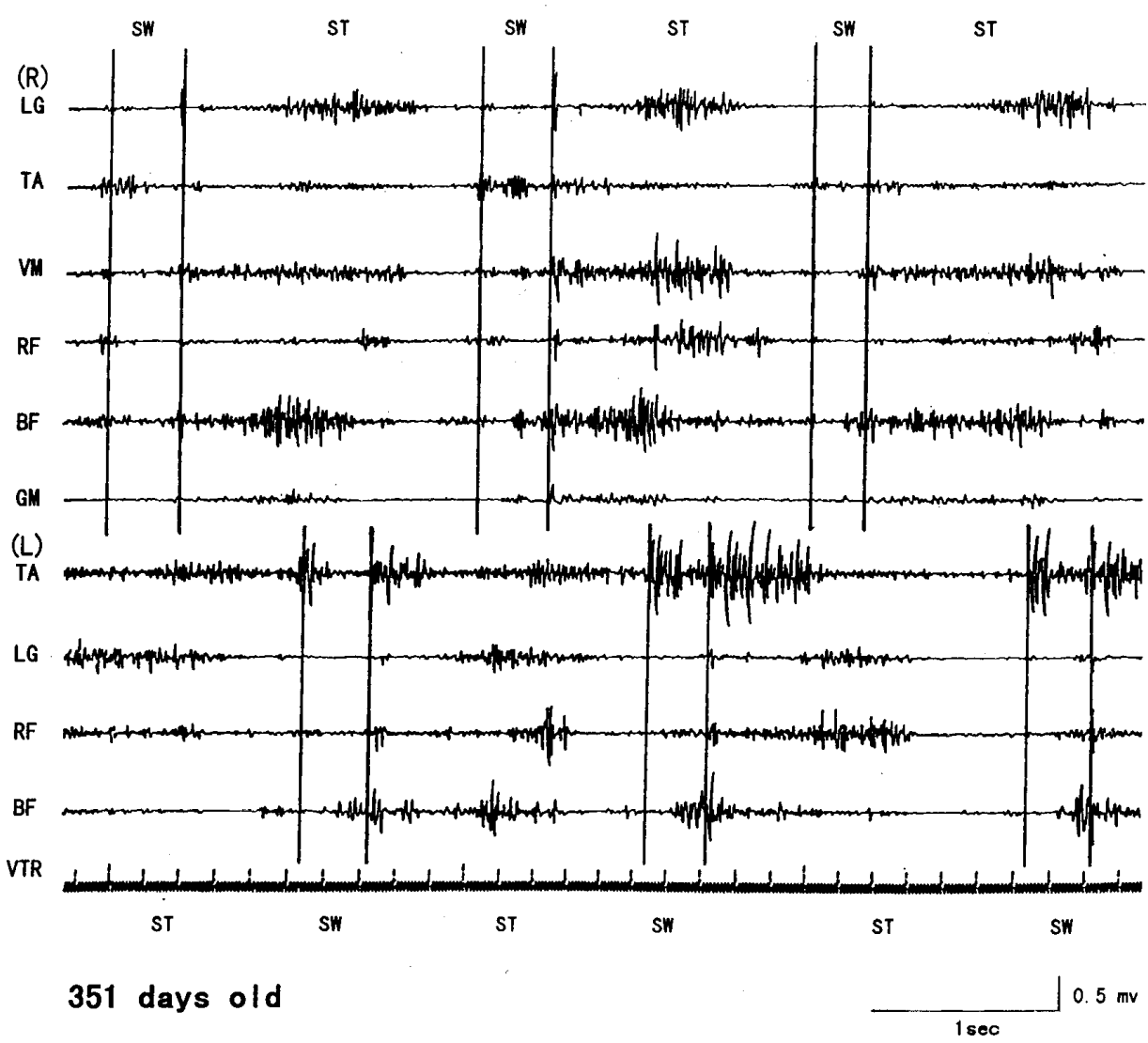

Fig. 5. EMGs of supported walking at 351 days after birth, 34 days before independent walking (same subject as in Figs. 1, 2, 3, and 4). Abbreviations are the same as those in Fig. 2.

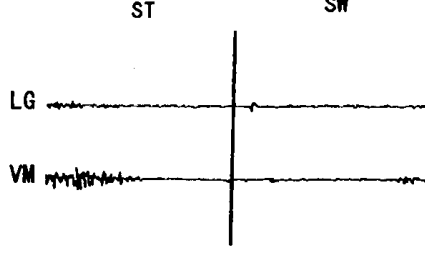

14 days old
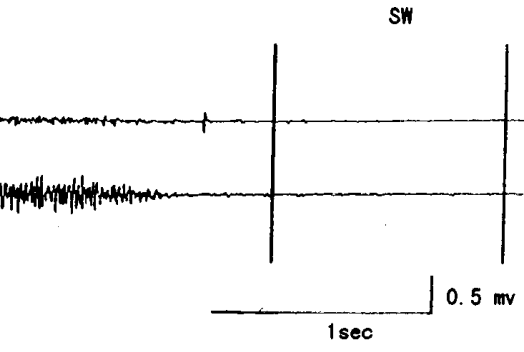

ST

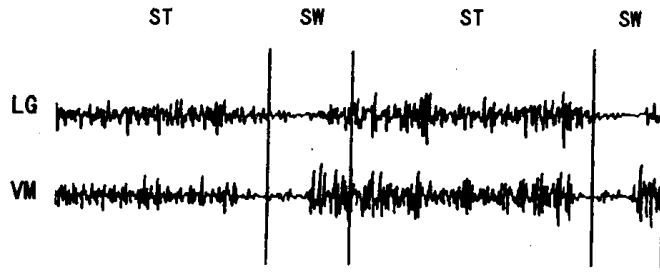

83 days old
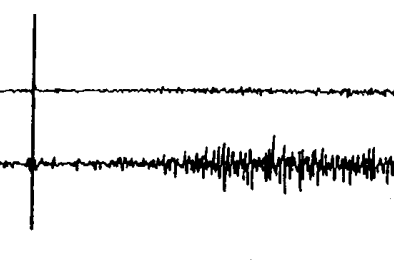

ST

ST

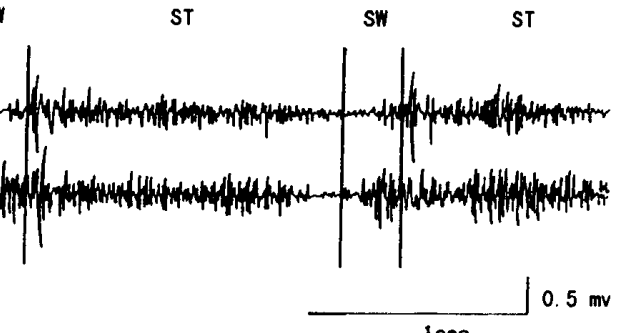

sec

Fig. 6. EMGs of newborn stepping at 14 and 83 days after birth (subject B). Abbreviations are the same as those in Fig. 2. 
Table 2. Activities of the lateral gastrocnemius (LG) and vastus medialis (VM) in the latter part of swing phase of stepping induced during very early development

\begin{tabular}{ccccc}
\hline \multirow{2}{*}{ Subject } & Muscle & \multicolumn{3}{c}{ Months after birth } \\
\cline { 3 - 5 } & & $0-1$ & $1-3$ & $3-4$ \\
\hline B & LG & $(-)$ & $(+),(-)$ & $(+)$, partly (-) \\
& VM & $(-)$ & $(+),(-)$ & $(+)$, partly (-) \\
C & LG & $(-)$ & $(-)$, partly $(+)$ & $(+),(-)$ \\
& VM & $(-)$ & $(+),(-)$ & $(+)$, partly (-) \\
D & LG & $(-)$ & $(-)$, partly $(+)$ & $(+),(-)$ \\
& VM & $(-)$ & $(-)$, partly $(+)$ & $(+),(-)$ \\
E & LG & $(-)$ & $(+),(-)$ & $(+)$, partly (-) \\
& VM & $(-)$ & $(+),(-)$ & $(+)$, partly (-) \\
F & LG & $(-)$ & $(+),(-)$ & $(+)$, partly (-) \\
& VM & $(-)$ & $(+),(-)$ & $(+)$, partly (-) \\
\hline
\end{tabular}

$(+)$ : noticeable activity, $(-)$ : no activity, $(+),(-)$ : instances of noticeable activity and of no activity intermingled.

TA and RF accompanied by greater activity in the LG and BF may have been due to forward leaning of the body ${ }^{3,5,19)}$. When, conversely, activity in the $\mathrm{LG}$ and $\mathrm{BF}$ decreased or disappeared while that in the TA and RF increased, this may have resulted from leaning backward ${ }^{5}$. Cocontraction of these muscles was also observed in many instances, probably related to maintaining a standing posture with the body erect or to stabilizing of the ankle, knee, and hip joints ${ }^{3,5,7)}$. These variations of leg muscle activities may be caused by the changing posture ${ }^{3)}$ during supported newborn stepping.

During stepping, sustained discharges of the VM are probably attributable to bearing body weight with the knee flexed ${ }^{3,5,7)}$. When VM activity was low or absent during stance phase, the manner of bearing weight on the knee joint may have resulted in a smaller load. We thus suggest that the degree of activity in the VM may be regarded as an indication of magnitude of load on the knee joint ${ }^{3,5,7)}$.

Even if the EMG patterns of leg muscles in stance phase appear to indicate unstable walking during stepping, we hesitate to consider them as reliably consistent signs of instability because variations in discharge patterns of the leg muscles are probably closely related to magnitude of joint load, which can be influenced by many factors other than instability. We thus find it undesirable to apply the EMG index of gait instability to discharge patterns in stance phase, during which
Table 3. Activities of the lateral gastrocnemius (LG) and vastus medialis (VM) in the latter part of swing phase of stepping during the first year of development in subject $\mathrm{A}$

\begin{tabular}{ccccc}
\hline \multirow{2}{*}{ Muscle } & \multicolumn{4}{c}{ Months after birth } \\
\cline { 2 - 5 } & $0-1$ & $1-3$ & $3-4$ & $6-12$ \\
\hline LG & $(-)$ & $(+),(-)$ & $(+)$, partly (-) & $(-)$, partly (+) \\
VM & $(-)$ & $(+),(-)$ & $(+)$, partly (-) & $(-)$, partly (+) \\
\hline
\end{tabular}

$(+)$ : noticeable activity, $(-)$ : no activity, $(+),(-)$ : instances of noticeable activity and of no activity intermingled.

considerable variations are seen in newborn stepping.

Secondly, we focus on developmental changes in the EMG patterns of supported walking during swing phase. Up to the first month of age, muscular activities of the LG and VM were hardly seen in the latter part of swing phase (Fig. 2, 6-top, Tables 2, 3), as also reported by Thelen et al. ${ }^{2}$ and Yamashita et al. ${ }^{19)}$. The leg extends passively ${ }^{17,}$ 19) and the foot contacts the floor usually with the heel first ${ }^{19)}$, as mentioned in our current results. These findings clearly show that muscular activities for knee extension and ankle plantarflexion are not observed in this period ${ }^{2,17,19)}$. As mentioned above (Table 1), Okamoto et al.5) determined that activity of the leg extensors before floor contact indicates gait instability, but it is risky to judge the presence of gait stability from an absence of activity in these two muscles in the neonatal period, because stepping in the neonatal period is a reflex movement performed under the control of lower (spinal) levels of the central nervous system (CNS) ${ }^{20)}$ and equilibrium reflexes are yet immature.

From one to three months, the leg extensors begin to show some activity before the foot reaches the floor (Fig. 2, 6-bottom, Table 2, 3) 2, 14, 15, 17-19) and the forefoot begins to contact the floor first more often. When the infant begins to actively perform knee extension and ankle plantarflexion, the strong muscle activities of leg extensors begin to participate before floor contact in this period. The marked discharge patterns of these two muscles are similar to those of unstable supported walking ${ }^{3)}$ and very unstable independent walking for the first time $3,5-9,22$ ). From these findings, we believe that the activities of the leg extensors before floor contact in this period indicate lack of stability, as identified by Okamoto et al.5) and 
Yamashita et al. ${ }^{19)}$ We thus believe that infants in this period begin to feel instability.

From around three to four months old, marked activity of the leg extensors are observed (Tables $2,3)^{19}$. Active ankle plantarflexion and knee extension before foot contact become the mode of performance in this period. Milani-Comparetti ${ }^{23)}$ reported that the parachute reaction of the legs begins to appear at about four months after birth. The parachute reaction is an equilibrium reflex performed under control of higher (cortical) levels of the $\mathrm{CNS}^{20)}$. Strong muscle activities of the leg extensors before floor contact may arise as parachute reactions (self-protection) with the maturation of the CNS. Although McGraw ${ }^{12)}$ pointed out that it is difficult sometimes to tell whether the active leg extension before floor contact is deliberate or of reflex quality, we would suggest that it is the beginning of voluntary infant stepping (supported walking) added upon reflex neonate stepping at this period.

From six to twelve months, absence of activity in these muscles before floor contact becomes the rule, and marked activity tends to disappear in the remaining instances (Table 3) ${ }^{3,7,19)}$. Lack of activity in these muscles indicates that leg extension before foot contact is not performed actively in this period, in contrast with the active leg extension of around three or four months. The developmental change of infant stepping in this period is due mainly to maturation of the equilibrium system and development of strength ${ }^{2}$. We interpret the absence of activity in the leg extensors in the latter part of swing phase in this period to resemble adult stable walking and thus to have resulted from development of strength, balance, and postural control $^{2)}$.

\section{CONCLUSION}

We made longitudinal observations on six normal neonates to see whether an EMG index of gait instability derived from the developmental process of normal infant walking is applicable to EMG patterns of supported walking in neonates and infants.

Muscular activities of the LG (an ankle plantarflexor) and VM (a knee extensor) in the latter part of swing phase indicate unstable walking, findings which are applicable to developmental changes during newborn stepping and infant supported walking.
In stepping during the first month, muscular activities were not seen in LG or VM. It would be misleading to consider such gait to be "stable" simply because these muscles are inactive in the neonatal period, especially since stepping in the neonatal period is under the control of lower levels of the central nervous system.

At around the third postnatal month, LG and VM showed strong activity just before the foot reached the floor, suggesting that muscular activities participating in active ankle plantarflexion and knee extension act as a parachute reaction. This may be the beginning of superimposition of voluntary infant stepping on top of reflex neonate stepping.

From six to twelve months, when the infant was becoming able to maintain standing without support, marked activities of the LG and VM before floor contact tended to disappear as in the adult pattern. We thus presume that absence of activity in the LG and VM at that time suggests stable walking, reflecting development of strength, balance and postural control during that period.

In summary, our observations of developmental changes in newborn stepping and infant supported walking, combined with an EMG index of gait instability based on subsequent stages of development, lead us to believe that activities of the LG and VM in the latter part of swing phase can be interpreted in terms of a scheme of early development of stability in walking.

\section{Acknowledgments}

Thanks are due to Dr. Y. Goto (Hyogo University of Teacher Education) and Mrs. H. Tsutsumi (Kansai Medical University) for their cooperation.

\section{REFERENCES}

1) Thelen E, et al: The developmental origins of locomotion. In: Woollacott MH, Shumway-Cook A (eds), Development of Posture and Gait Across the Life Span. University of South Carolina Press, South Carolina, 1989, pp 25-47.

2) Thelen E, et al: Relationship between newborn stepping and later walking: A new interpretation. Develop Med Child Neurol 29: 380-393, 1987.

3) Okamoto T, et al: Human infant pre-independent and independent walking. In: Kondo S. (ed), Primate Morphophysiology, Locomotor Analyses and Human Bipedalism. University of Tokyo Press, Tokyo, 1985, pp 25-45.

4) Okamoto K, et al: Electromyographic case study of recovery of walking in an elderly man after cerebral 
infarction. J Phys Ther Sci 10: 19-25, 1998.

5) Okamoto K, et al: An index of gait instability based on electromyographic findings during the development of gait. J Phys Ther Sci Sci 10: 61-71, 1998.

6) Okamoto $T$, et al: Electromyographic study of the learning process of walking in infants. Electromyography 12: 149-158, 1972.

7) Kazai N, et al: Electromyographic study of supported walking in infants in the initial period of learning to walk. In: Komi PV. (ed), Biomechanics V-A. University Park Press, Baltimore, 1976, pp 311-318.

8) Okamoto T, et al: Electromyographic study of the bifunctional leg muscles during the learning process in infant walking. In: Matsui H, Kobayashi K. (eds), Biomechanics VIII-A. Human Kinetics Publishers, Illinois, 1983, pp 419-422.

9) Okamoto K, et al: Electromyographic characteristics of the learning process of walking in normal infants and children. In: Yabe K, Kusano K, Nakata H (eds), Adapted Physical Activity: Health and Fitness. Springer-Verlag, Tokyo, 1994, pp 137-141.

10) Basmajian JV, et al: Muscles Alive: Their Functions Revealed by Electromyography, 5th ed. Williams \& Wilkins, Baltimore, 1985.

11) Winter DA: The Biomechanics and Motor Control of Human Gait, 2nd ed. University of Waterloo Press, Ontario, 1991.

12) McGraw MB: Neuromuscular development of the human infant as exemplified in the achievement of erect locomotion. J Pediat 17: 747-771, 1940.

13) Thelen E: Learning to walk: Ecological demands and phylogenetic constraints. In: Lipsitt LP (ed), Advances in Infancy Research 3. Ablex, Norwood NJ, 1984, pp 213-250.
14) Forssberg H: Ontogeny of human locomotor control: I. Infant stepping, supported locomotion and transition to independent locomotion. Exp Brain Res 57: 480-493, 1985.

15) Forssberg H: Development and integration of human locomotor functions. In: Goldberger ME, Gorio A, Murray M (eds), Development and Plasticity of the Mammalian Spinal Cord. Fidia Research Series III. Liviana Press, Padova, 1986, pp 53-63.

16) Zelazo PR, et al: "Walking" in the newborn. Science 176: 314-315, 1972.

17) Thelen E, et al: Newborn stepping: An explanation for a "disappearing" reflex. Dev Psychol 18: 760775, 1982.

18) Forssberg $\mathrm{H}$, et al: Infant locomotion: A preliminary movement and electromyographic study. In: Berg K et al (eds), Children and Exercise IX. University Park Press, Baltimore, 1980, pp 32-40.

19) Yamashita H, et al: Electromyographic study of infant primitive walking. Proceedings of International Council for Health, Physical Education and Recreation 36: 509-514, 1993.

20) Shoji J: The development of reflexes in early infancy. J Child Develop 12: 11-23, 1976.

21) Okamoto T, et al: A simple procedure to attenuate artifacts in surface electrode recordings by painlessly lowering skin impedance. Electromyogr Clin Neurophysiol 27: 173-176, 1987.

22) Sutherland $\mathrm{DH}$, et al: The development of mature gait. J Bone Joint Surg 62-A (3): 336-353, 1980.

23) Milani-Comparetti A, et al: Routine developmental examination in normal and retarded children. Develop Med Child Neurol 9: 631-638, 1967. 